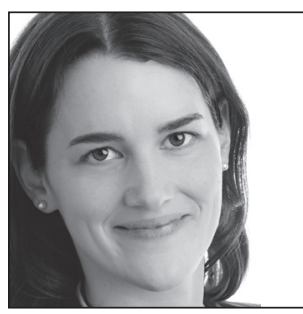

Doris Forster

Professeure associée für Römisches Recht und Privatrechtsgeschichte

Universität Genf

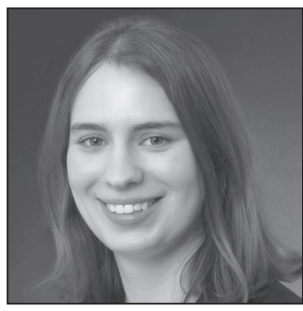

Janika Rieder

Universität Konstanz

\title{
Roboter als Rechtssubjekte - Der Streit um die E-Person
}

\section{Der Mensch und autonome Agenten}

Die rasanten Fortschritte bei der Entwicklung künstlicher Intelligenz haben sog. autonome Agenten längst in den Fokus der juristischen Debatten gerückt. Autonom agierende Maschinen, die über Intelligenz verfügen, das heißt lernen und ihre Verhaltensregeln dadurch selbst ändern können, sollen in der Zukunft menschenähnlich mit der Fähigkeit ausgestattet sein, „Entscheidungen zu treffen und diese in der äußeren Welt unabhängig von externer Steuerung oder Einflussnahme umzusetzen“. ${ }^{* 1}$ Anders als bei den bisher lediglich automatisierten Systemen, der schwachen KI, wären die Entscheidungen und das daraus resultierende Verhalten der Maschine mit starker KI dann aufgrund der Komplexität der zugrundeliegenden Algorithmen nicht unbedingt vorhersehbar und demzufolge nicht mehr steuerbar.

Die technische Transformation führt dazu, dass die gesellschaftliche Trennlinie zwischen Mensch und Maschine mitunter verwischt. Bereits seit über einem Jahrhundert ist die Vermenschlichung von Maschinen ein beliebter Topos in Film, Kunst und Kultur. ${ }^{{ }_{2}}$ Technikerinnen und Konstrukteure benutzen ihre Phantasie, um diese möglichen künstlichen Agenten humanoid zu gestalten, Maschinen erhalten menschliche Stimmen und Namen wie Alexa; Roboter stecken in der Hülle menschenähnlicher Körper. Die Philosophie hinterfragt, inwieweit es „künstliche Agenten“ überhaupt geben kann: Können Maschinen selbstbestimmt denken und handeln, können sie als autonome Subjekte geradezu menschenähnlich oder analog einem Tier gesehen werden? Die Rechtswissenschaft begegnet wirtschaftspraktischen Bedürfnissen: Wer zahlt für die durch diese Agenten neu entstehenden Risiken und Schäden? Und auch hier scheinen die Grenzen zwischen Mensch und Maschine zu verschwimmen, wenn teils als Novum vorgeschlagen wird, die Maschine selbst zum Haftungssubjekt zu machen und ihr die Qualität eines Rechtssubjekts zu verleihen. So hat 2017 das Europäische Parlament die Europäische Kommission aufgefordert, in einer neuen Robotik Richtlinie autonomen Robotern den Status einer E-Person zu geben. ${ }^{*} 3$

Diese Entwicklung gibt Anlass, den Rechtsubjektbegriff und die Einordnung der durch die starke KI entstehenden autonomen Agenten de lege lata und de lege ferenda zu überprüfen. Der Begriff des (autonomen) Roboters wird vorliegend im Sinne einer autonom handelnden Maschine mittels starker KI verwendet. ${ }^{*}$

1 Europäisches Parlament, „Zivilrechtliche Regelungen im Bereich Robotik“ (Entschließung), P8_TA (2017) 0051 AA.

2 Der Name „Roboter“ stammt aus dem tschechischen Theaterstück „R.U.R.“ von 1921. Die Bezeichnung war ein zufälliger Einfall des Bruders des Machers, Josef Ĉapek, für eine intelligente Maschine, der sich von dem tschechischen Ausdruck „robota“ für Fronarbeit ableitet: Svetlana Efimova, „Der ,Roboter“ wird 100. Über eine (nicht nur) literarische Utopie.“ <https:// geschichtedergegenwart.ch/der-roboter-wird-100-ueber-eine-nicht-nur-literarische-utopie/> abgerufen am 11.02.2021.

3 Ibid.

4 Ein einheitliches Begriffsverständnis für KI ist bis heute nicht erreicht: Martin Ebers, in Martin Ebers and others (Hrsg.) Künstliche Intelligenz und Robotik (C.H. Beck 2020) § 3, Rn. 4 - 7. - DOI: https://doi.org/10.17104/9783406769818; Susanne Beck, „Grundlegende Fragen zum rechtlichen Umgang mit der Robotik“ (2009) JR 225, 226. - DOI: https://doi. 


\section{Potentielle Lückenschließung im Zivilrecht durch die E-Person}

Die geltenden Regeln des BGBs wurden im analogen Zeitalter geschaffen. Eine Lücke im geltenden Recht könnte die E-Person im Bereich des Vertragsschlusses und der Haftung füllen. Schon heute wird eine Vielzahl von Rechtsgeschäften ohne menschliche Beteiligung abgeschlossen. Beim „Hochfrequenzhandel“ bestimmt ein Algorithmus über Zeitpunkt, Anzahl und Volumen des Auftrags und ob ein Mensch ausnahmsweise den Auftrag übernehmen soll. ${ }^{*}$ Eine „Maschinenerklärung“ kennt das BGB hingegen nicht. Eine Willenserklärung setzt grundsätzlich menschliches (Vor)Verhalten voraus. Mögliche Anknüpfungspunkte nach dem BGB für die Zurechnung des autonomen Roboterhandelns wären lediglich eine Botenstellung des Roboters, doch mangelt es hierfür schon an einer fremden Willenserklärung, wenn der Roboter selbst die Erklärung erzeugt. ${ }^{* 6}$ Stuft man den Roboter als Stellvertreter ein, ${ }^{*}{ }^{7}$ wird das Festlegen des Vertretungsumfangs problematisch, da der Roboter sein Handeln selbst bestimmt. Überschreitet er die Vertretungsmacht, entsteht in Anbetracht einer fehlenden Haftungsmasse eine Haftungslücke nach § 179 BGB. ${ }^{* 8}$ Im Rahmen der Zurechnung nach den allgemeinen Grundsätzen der Rechtsgeschäftslehre stellt im Einzelfall ein sehr hoher Grad an Autonomie die Zurechnung der Willenserklärung zum Verwender in Frage. ${ }^{* 9}$

Würde die Maschine über eine Subjektqualität verfügen, wäre sie selbst der eigentliche Urheber einer „Maschinenerklärung“, eine mit den Regeln des BGB nur mühsam zu bewältigende Zurechnung wäre nicht mehr erforderlich. Die Maschine würde aus dem Vertrag berechtigt und verpflichtet, was allerdings wiederum zu komplexen Folgefragen führen würde.

Im Bereich des geltenden Haftungsrechts stellt sich bei der Produkthaftung die Frage, ob ein lediglich aus Software bestehender Roboter in den Anwendungsbereich des Produkthaftungsgesetzes fällt, wenn durch ihn Schäden entstehen. ${ }^{* 10}$ Besteht der Roboter aus mehreren Teilen einer zusammengesetzten Software, ergeben sich zudem Beweisprobleme für den Geschädigten beim Nachweis des Kausalverlaufs. ${ }^{* 11} \mathrm{Im}$ Rahmen einer Nutzerhaftung gem. § 823 I BGB zeigen sich Schwierigkeiten ein eigenes Verschulden des Nutzers zu begründen, da dieser bei autonom agierenden Robotern keine Einfluss- und Kontrollmöglichkeit hat. ${ }^{*}$ Auch ein Rückgriff auf die Gefährdungshaftungstatbestände, insbesondere auf die Tierhalterhaftung gem. § 833 BGB, stellt mangels Analogiefähigkeit der Gefährdungshaftung keine Alternative dar. ${ }^{* 13}$

org/10.1515/juru.2009.225; Maximilian Herberger, ,'Künstliche Intelligenz und Recht“ (2018) NJW 2825, 2826; Thomas Riehm und Stanislaus Meier, „Künstliche Intelligenz im Zivilrecht“ in Veronika Fischer, Peter Hoppen und Jörg Wimmers (Hrsg.), DGRI Jahrbuch 2018 (Verlag Dr. Otto Schmidt 2019), 1, 37. - DOI: https://doi.org/10.9785/9783504386757-002. Das Europäische Parlament hat daher auch zur Schaffung einer einheitlichen Begriffsdefinition aufgefordert: Europäisches Parlament (Fn 1).

5 Mario Martini, Blackbox Algorithmus - Grundfragen einer Regulierung Künstlicher Intelligenz (Springer 2019), 143. DOI: https://doi.org/10.1007/978-3-662-59010-2.

6 Luisa Specht und Sophie Herold, „Roboter als Vertragspartner? - Gedanken zu Vertragsabschlüssen unter Einbeziehung automatisiert und autonom agierender Systeme“ (2018) MMR 40, 43; Olaf Sosnitza, „Das Internet der Dinge - Herausforderung oder gewohntes Terrain für das Zivilrecht?“ (2016) CR 764, 766. - DOI: https://doi.org/10.9785/cr-2016-1124; Christiane Wendehorst und Julia Grinzinger in Martin Ebers (Fn 4) § 4, Rn. 32.

7 So Jan-Erik Schirmer, „Rechtsfähige Roboter?“ (2016) JZ 660, 664. - DOI: https://doi.org/10.1628/0022688 16x14615987983565.

$8 \quad$ Christiane Wendehorst und Julia Grinzinger (Fn 6) § 4, Rn. 32.

9 Peter Bräutigam und Thomas Klindt, „Industrie 4.0, das Internet der Dinge und das Recht“ (2015) NJW 1137, 1138; Malte Grützmacher und Jörn Heckmann, Autonome Systeme und KI - vom vollautomatisierten zum autonomen Vertragsschluss?“ (2019) CR, 553, 555. - DOI: https://doi.org/10.9785/cr-2019-350907; Gunther Teubner, „Digitale Rechtssubjekte? - Zum privatrechtlichen Status autonomer Softwareagenten“(2018) AcP, 155 179f. - DOI: https://doi.org/10.1628/acp-2018-0009. Die Vergleichbarkeit mit Blankett-Erklärungen ablehnend: Friedemann Kainer und Lydia Förster, „Autonome Systeme im Kontext des Vertragsrechts“" (2020) ZfPW 275, 290f.

10 Als Produkt i.S.d. § 2 ProdHaftG wird grundsätzlich ein beweglicher, körperlicher Gegenstand gefordert. Christian Förster in Beck'scher Onlinekommentar zum ProdHaftG (56. Aufl. 2020), § 2 Rn. 4; Gerhard Wagner, „Robot, Inc.: Personhood for Autonomous Systems?” (2019) 88 Fordham L. Rev. 591, 604. Für eine Anwendung auf bloße Software: Gerhard Wagner in Münchener Kommentar zum BGB, Band 7 (8. Auflage, C.H. Beck 2020), § 2 ProdHaftG, Rn. 26f.; Jan Eichelberger in Ebers (Fn 4) § 5, Rn. 45; a.A. Jürgen Oechsler in Staudinger (Sellier de Gruyter 2018), § 2 ProdHaftG, Rn. 65f.

11 Wagner (Fn 10) 603.

12 Ibid 607.

13 Die Analogiefähigkeit ablehnend bereits RGZ 78, 171 (172) sowie BGHZ 55, 229 (234) wegen des Ausnahmecharakters der Gefährdungshaftung. Ebenso Christian Förster in BeckOK zum BGB (56. Aufl. 2020), § 823 Rn. 78; Gerhard Wagner in MüKoBGB (Fn 10) Vorb § 823, Rn. 25f. aA Zulässigkeit der Einzelanalogie bei Johannes Hager, in Staudinger (Sellier de 
Wäre hingegen die Maschine das Haftungssubjekt, müsste sie für den durch sie verursachten Schaden einstehen. Die finanziellen Mittel kämen bspw. aus einer Pflichtversicherung oder aus einem Haftungsfonds in den der Produzent und der Betreiber einzahlten. ${ }^{*} 4$ Doch auch diese Lösung stellt kein Allheilmittel dar: in bestimmten Fällen, sei die „KI“ nicht zu lokalisieren, ein Umstand, der auch nicht durch ein Maschinenregister ohne weiteres beseitigt werden könne. Die Idee eines Haftungsfonds sei ebenfalls kein zuverlässiger Schutz des Geschädigten vor dem Insolvenzrisiko. ${ }^{*} 5$

\section{Umsetzungsmöglichkeiten des Rechtssubjektstatus für KI im BGB}

Für die Umsetzung des Rechtssubjektstatus von autonomen Robotern würden verschiedene Lösungen in Betracht kommen. Eine Möglichkeit ist es, den Roboter ausschließlich mit Geschäftsfähigkeit auszustatten. Doch führt die Integration dieses Ansatzes in das BGB zu grundlegenden Systembrüchen. Das BGB setzt die Rechtsfähigkeit voraus, um Geschäftsfähigkeit gewähren zu können. Wer kein Träger von Rechten und Pflichten ist, kann auch nicht wirksam Rechtsgeschäfte tätigen. ${ }^{*} 16$ Eine vollkommene Abstraktion von Rechts- und Geschäftsfähigkeit wie im römischen Recht ist dem BGB fremd. ${ }^{*}{ }^{17}$

Eine Alternative stellt die Verleihung der vollen Rechtsfähigkeit dar, sodass der Roboter umfassend mit Rechten und Pflichten ausgestattet wird. Damit werden Roboter und Menschen in ihrer Behandlung gleichgestellt. Das führt nicht nur aus moralischer Sicht zu Schwierigkeiten, sondern auch rechtspraktisch ergeben sich Folgeprobleme: Soll ein Roboter auch das Recht zur Heirat oder Erbfähigkeit bekommen? Bedarf er des Schutzes vor körperlichen Schmerzen, wenn die KI keinen biologischen Körper hat? ${ }^{* 18}$ Es zeigt sich: Eine absolute Gleichstellung von Mensch und KI ist weder erforderlich noch würde sie der anthropozentrischen Ausrichtung des Grundgesetzes gerecht werden. An dieser Stelle muss daher zwischen Mensch und Roboter differenziert werden. Zwar soll der Roboter eingeschränkt Rechte und Pflichten erhalten, die zu einer Verbesserung des Umgangs mit Robotern im rechtlichen Alltag führen. Dies ist notwendig, da der Roboter kein lebloses Objekt ist, sondern eigenständig kommuniziert und agiert. Allerdings geht die Gewährung von vollständiger Rechtsfähigkeit zu weit.

Eine weitere Möglichkeit wäre, eine neue Gesellschaftsform in Form einer „E-GmbH“ zu gründen. ${ }^{{ }^{*} 19}$ Diese haftet, verbunden mit einem Haftungsfonds, für Schäden, die der Roboter verursacht. Vorteilhaft an dieser Lösung ist, dass sich die Debatte, ob ein Roboter eine Rechtspersönlichkeit erhalten kann, nicht stellt, sondern dass vielmehr mit den bestehenden Konzepten der natürlichen und juristischen Person gearbeitet werden kann. Allerdings ist diese Lösung lediglich eine dogmatische Konstruktion, um den Roboter nicht an die Subjektstellung heranzurücken. Anstatt die Haftung direkt an den Roboter zu knüpfen, wird ein künstlicher Umweg über eine elektronische Gesellschaft gewählt. Dieser führt zu einer erhöhten Komplexität anstelle einer Vereinfachung, da sich zusätzlich gesellschaftsrechtliche Probleme ergeben.

Gruyter 2017), Vorb § 823, Rn. 29. Herbert Zech, „Künstliche Intelligenz und Haftungsfragen“ (2019) ZfPW 198, 214f hält de lege lata § 833 BGB analog für KI für möglich, lehnt diese aber wegen § $833 \mathrm{~S}$. 2 BGB und der mangelnden Beherrschbarkeit von KI bei privaten Nutzer im Ergebnis ab. De lege ferenda ist § 833 BGB als Alternative umstritten. Zustimmend Peter Bräutigam und Thomas Klindt (Fn 9), 1139; Olaf Sosnitza (Fn 6) 772; zurückhaltend Susanne Horner und Markus Kaulartz, „Haftung 4.0. Rechtliche Herausforderungen im Kontext der Industrie 4.0“ (2016) InTeR, 22, 24. Ablehnend jedoch: Georg Borges, „Rechtliche Rahmenbedingungen für autonome Systeme“ (2018) NJW 977, 981; Malte Grützmacher, „Die deliktische Haftung für autonome Systeme - Industrie 4.0 als Herausforderung für das bestehende Recht“ (2016) CR, 695, 698. - DOI: https://doi.org/10.9785/cr-2016-1015.

14 Susanne Beck „Über den Sinn und Unsinn von Statusfragen - zu Vor- und Nachteilen der Einführung einer elektronischen Person“ in Eric Hilgendorf (Hrsg.) Eric Hilgendorf (Hrsg.), Robotik und Gesetzgebung (Nomos Verlag 2013), 256; Europäisches Parlament (Fn 1) AI. 58.

15 Martin Ebers (Fn 4) § 3 Rn. 76-80.

16 Steffen Wettig und Eberhard Zehender, „A legal analysis of human and electronic agents“ (2004) Artif Intell Law, 111, 123f. - DOI: https://doi.org/10.1007/s10506-004-0815-8.

17 Ibid 127.

18 Susanne Beck (Fn 14) 255.

19 So ein Vorschlag von Christiane Wendehorst, „Ist ein Roboter haftbar?“ (2020), 1 Forschung und Lehre. Der Lösungsweg über das Gesellschaftsrecht findet sich auch hinsichtlich amerikanischer Gesellschaften (LLC) bei Shawn Bayern, „The Implications of Modern Business-Entity Law for the Regulation of Autonomous Systems“ (2015) 19 Stan. Tech. L. Rev. 93. 
Vor dem Hintergrund der fortschreitenden Entwicklung der Technik erscheint es sinnvoll, neue Regelungen zu schaffen, die an den Roboter selbst anknüpfen, anstatt den Roboter in bestehende Konstruktion zu „pressen“, um auch auf zukünftig auftretende Fragen flexibler reagieren zu können. In diesem Sinne wäre der Ansatz, den auch das Europäische Parlament gewählt hat, sinnvoll: die Schaffung eines Sonderstatus der elektronischen Person. ${ }^{{ }_{2} 0}$ Diese Lösung ließe sich systematisch in das BGB integrieren, indem der zweigliedrige Aufbau der Rechtssubjekte (natürliche und juristische Personen) um eine dritte Kategorie (elektronische Person) erweitert wird. Die Schaffung einer Sonderkategorie hätte den Vorteil, dass die bisherigen Kategorien nicht neu interpretiert oder erweitert werden müssen. Die neue Kategorie werde stattdessen spezifisch auf die Probleme im Umgang mit Robotern zugeschnitten. ${ }^{* 21}$ Die Teilrechtsfähigkeit des Roboters geht nur soweit, wie dies sinnvoll und erforderlich ist. Der Roboter wird also spezifisch mit Rechten und Pflichten ausgestattet, die notwendig für den Umgang mit Robotern im Rechtsverkehr sind. ${ }^{{ }_{22}}$ Auf diese Art könnte eine eigene Roboterhaftung für durch den Roboter verursachte Schäden geschaffen werden. In den Fällen, bei denen eine menschliche Haftung ausgeschlossen ist, wird so eine Haftungslücke vermieden. ${ }^{* 23}$ Zusätzlich könnte der Roboter mit Geschäftsfähigkeit ausgestattet werden, sodass er wirksam rechtsgeschäftlich handeln kann, um die Probleme beim Vertragsschluss zu lösen.

\section{Maschinen als Rechtsubjekte}

Unabhängig davon, ob die E-Person eine sinnvolle vertrags- oder haftungsrechtliche Lösung bietet, stellt sich jedoch die grundlegende Frage, ob eine Erweiterung des Rechtssubjektbegriffs auf Maschinen rechtlich zulässig ist oder aber bspw. eine verfassungsrechtliche Unzulässigkeitsentscheidung sie verhindere. Verliert der Mensch als herausgehobenes, würdetragendes Rechtssubjekt seine exklusive Stellung, könnten Kritiker und Kritikerinnen dies als Paradigmenwechsel und Vorstufe zum „Posthumanismus“ verwerfen.

\subsection{Rechtshistorische Sicht}

Die Einordnung von Maschinen als Rechtssubjekte mag aus der heutigen Sicht befremden, da in unserer Rechtsordnung einzig der Mensch als frei denkendes und moralisch handelndes Individuum als Träger von Rechten und Pflichten anerkannt wird. Ein Blick in die antike Rechtsgeschichte zeigt die zeitliche Wandelbarkeit dieser Annahme.

Grenzüberschreitungen und -verwischungen zwischen dem Menschen und nichtmenschlichen Lebewesen finden sich auch in der griechischen Antike. Hier sind es nicht die humanoiden Maschinen, es sind anthropomorphe Tiere, zu denen sich der Mensch in Beziehung setzt, andererseits abgrenzt.

Aristoteles entwarf ein sehr konkretes Tierbild: Er billigte den höher entwickelten Tieren eine Art Verstandeskraft zu (phrónesis), mit der sie aus Erfahrungen lernen und sich situationsgerecht verhalten können. Sie müssten also Gedächtnis, Vorstellungskraft und Entschlussfähigkeit besitzen. Die Sonderstellung des Menschen rechtfertigt Aristoteles durch seine besondere Geisteskraft (noûs): Sie ermögliche allein dem Menschen an der göttlichen Vernunft teilzuhaben, Überlegungen anzustellen und rational zu planen. ${ }^{* 24}$ In der griechischen Antike wurde anerkannt, dass Tiere lernfähig sind und ihr Verhalten dementsprechend anpassen können. ${ }^{* 25}$ Das Tierbild steht damit der heutigen Definition von Autonomie auffällig nahe. Der Umgang mit Tieren im Recht und vor allem im Prozess war ein gänzlich anderer als heute. Im antiken Griechenland waren Tierprozesse üblich. In Athen wurden Haustiere bestraft, wenn sie für die Götter bestimmte Opfergaben anrührten. ${ }^{* 26}$ Ein Gesetz auf Salamis bestimmte, dass einem Schwein, das fruchttragende Felder verwüstete,

20 Europäisches Parlament (Fn 1) AI. 59.

21 Susanne Beck (Fn 14) 255.

22 Gunther Teubner (Fn 9) 182.

23 Gregor Fitzi, „Roboter als „legale Personen“ mit begrenzter Haftung. Eine soziologische Sicht“, in Eric Hilgendorf (Hrsg.) (Fn 14) 393. - DOI: https://doi.org/10.5771/9783845242200-377.

24 Thomas Macho, „Einführung“ in Hartmut Böhme (Hrsg.), Tiere: Eine andere Anthropologie, Band 3 (Böhlau 2004), $73,75$.

25 Aristoteles, Historia animalium I.1.488b, 24-27; Martin F. Meyer, „Aristoteles über die Natur des Menschen“ in Marko J Fuchs und Annett Wienmeister (Hrsg.), Funktion und Normativität bei Darwin und Aristoteles, Band 23 (University of Bamberg Press 2016), 79, 103

26 Plutarch, de sollertia animalium, c 2. 
zur Strafe die Zähne ausgebrochen werden. ${ }^{{ }^{2} 27}$ Auf der anderen Seite wurden Tiere für gute Taten auch öffentlich belohnt. ${ }^{* 28}$ Tiere waren als Parteien im Prozess vertreten. Sie konnten in den Tierprozessen öffentlich angeklagt werden und wurden in diesem Sinne als Rechtssubjekte behandelt. ${ }^{* 29}$

Für das archaische römische Recht ist nicht eindeutig belegt, ob es wie in Griechenland Tierprozesse gab. Teils wird in der Literatur angenommen, etwa noch von Mommsen oder aktueller Kaser, dass ursprünglich im römischen Recht bei Tierschäden das Tier als Täter angesehen wurde. Immer wenn das Tier einen Schaden verursacht habe, sei es der ihm innewohnende Tierdämon, der dafür verantwortlich sei. ${ }^{*}{ }^{30}$ Diese archaischen Vorstellungen vom Tier als Täter und Tierdämonen wurden in der römischen Klassik überwunden. Die klassische römische Jurisprudenz fand mittels der actio de pauperie (Klage aus Tierschaden) einen beeindruckenden Ausgleich zwischen Tradition einerseits und einem Haftungsrecht, das sich durch eine Verantwortungsallokation beim Tiereigentümer im Sinne eines effektiven Opferschutzes auszeichnet andererseits. Im klassischen römischen Recht finden wir kaum aussagekräftige Hinweise auf eine Subjektivierung der Tiere. ${ }^{*}{ }^{1}$ Tierprozesse sind allerdings nicht in der Antike ausgestorben. Sie waren im Mittelalter und in der frühen Neuzeit in Europa weit verbreitet. Kirchliche Prozesse richteten sich gegen Herden wilder Tiere oder solcher Arten, die gemeinhin als Plagen oder Schädlinge klassifiziert wurden, wie Ratten, Mäuse, Maulwürfe, Raupen, Schnecken, Schlangen, Kröten und Läuse. Diese Prozesse wurzelten in der Vorstellung, dass diese Tiere von Dämonen besessen wären. Die säkularen Prozesse in dieser Zeit kamen zur Anwendung, wenn ein Tier einen Menschen getötet oder verletzt hat. Erste Kritiker wie Philipp de Beaumanoir merkten an, dass die Bestrafung des Tieres durch die öffentliche Hinrichtung sinnlos sei, da das Tier keinen Verstand habe, um es als spezifische Bestrafung für sein Handeln zu begreifen. ${ }^{*}{ }^{32}$

In der griechischen Antike gab es nicht nur Tierprozesse. Auch gegen Sachen wie Flüsse und Säulen wurden Prozesse geführt. Wurde ein Mensch z.B. durch einen herabfallenden Gegenstand getötet, so wurde der Prozess gegen den Gegenstand geführt und der Gegenstand außer Landes gebracht. ${ }^{*} 3$ Im ionischen Thasos wurde ein Mensch durch eine umfallende Säule getötet. Die Hinterbliebenen des Opfers führten sodann einen Prozess gegen die Säule. ${ }^{*}{ }^{4}$ Ein anderes Beispiel ist ein Verfahren gegen eine Naturgewalt: im jonischen Kleinasien kam es durch einen Fluss zu Überschwemmungen, woraufhin die geschädigten Grundbesitzer sodann einen Prozess gegen den Fluss führten. Die Geldstrafe, die der verurteilte Fluss zahlen musste, wurde aus den Überfahrtgeldern finanziert. ${ }^{*} 35$ Aristoteles erklärte den Gegenstand, der den Erfolg verursacht hat, für verantwortlich, wenn ein menschliches Verschulden ausgeschlossen war. Der Gegenstand musste zur Verantwortung gezogen werden. ${ }^{*}{ }^{36}$

Zur Zeit Roms waren Sachprozesse ausgestorben. Der Fall der Grenzverletzung mit einem Pflug, welcher verlangte, den Täter samt Pfluggespann den Göttern zu opfern, stellte einen Einzelfall dar und

27 Aelianus, de natura animalium, 5, 45.

28 Rudolf Düll, „Zum Anthropomorphismus im antiken Recht“ (1944) ZRG 346, 347. - DOI: https://doi.org/10.7767/ zrgra.1944.64.1.346.

29 Rudolf Düll, „Archaische Sachprozesse und Losverfahren“ (1941) ZRG 1-10. - DOI: https://doi.org/10.7767/ zrgra.1941.61.1.1.

30 Max Kaser, Das Römische Privatrecht, Erster Abschnitt (2. Auflage 1971), 165. So auch: Heinrich Honsell, Römisches Recht (7. Auflage, Springer 2010), 164. - DOI: https://doi.org/10.1007/978-3-642-05307-8. Wolfgang Lorenz, Die Gefährdungshaftung des Tierhalters nach § 833 S. 1 BGB - Die funktionale Struktur der Gefährdungshaftung für die Risikoverteilung im Tierschadensrecht (Duncker und Humblot 1992), 66. - DOI: https://doi.org/10.3790/978-3-428-47470-7.

31 Zur actio de pauperie: Milena Polojac, Actio de pauperie and liability for damage caused by animals in Roman law (Dosije 2003); Maria V Giangrieco Pessi, Ricerche sull'Actio de pauperie: Dalle XII tavole ad Ulpiano Vol. 45 (Casa editrice dott. Eugenio Jovene 1995).

32 Rapahel Sealey, „Aristotle, Athenaion Politeia 57.4: Trial of Animals and Inanimate Objects for Homicide“ (2006) The Classical Quarterly 475, 481-482. - DOI: https://doi.org/10.1017/s0009838806000474.

33 Demosthenes 23,76.

34 Paul S Bermann, „An Anthropological Approach to Modern Forfeiture Law: The Symbolic Function of Legal Actions Against Objects" (1999) Yale Journal of Law \& the Humanities 1, 22; - DOI: https://doi.org/10.2139/ssrn.159329; Rudolf Düll (Fn 29) 13.

35 Strabon 12,8,19. Eine moderne Parallele stammt aus Neuseeland. Dort wurde am 20. März 2017 der Te Awa Tupua (Whanganui River Claims Settlement) Act 2017 erlassen. Darin wird Te Awa Tupua - darunter wird der Whanganui River sowie seine physischen und metaphysischen Elemente verstanden - in den Status einer „legal person“, also eines Rechtssubjekts, gehoben mit den daraus resultierenden Rechten und Pflichten. In der Rechtspraxis wird der Fluss als eine Art juristische Person behandelt. Hintergrund dieser Entscheidung war der Schutz der Rechte der dort ansässigen Maori Stämme, für die der Whanganui River ihr Lebensraum darstellt. Erin O’Donnell und Julia Talbot-Jones, „Creating legal rights for rivers: lessons from Australia, New Zealand, and India” (2018) Ecology and Society, 1.

36 Aristoteles, Athenaion politeia, 57, 4. 
entspricht nicht einem Sachprozess im strengen Sinne. Die Vorschrift hat sakralen Charakter und wird den frühen römischen Königsgesetzen zugeordnet. ${ }^{*}{ }^{37}$

Auf der anderen Seite zeigt uns die rechtshistorische Perspektive, dass nicht zwangsläufig jeder Mensch stets als Rechtssubjekt behandelt wurde. So wurden Sklaven im römischen Recht als Sachen klassifiziert. Sie waren nicht rechts- und vermögensfähig, sie konnten grundsätzlich keine Verträge in ihrem eigenen Namen abschlieBen, für ihre Delikte haftete ihr dominus, der Sklaveneigentümer (sog. Noxalhaftung). Ein Grundsatz, der für sämtliche alieni iuris, also auch Hauskinder, galt. Alieni iuris hatten grundsätzlich kein eigenes Vermögen, weshalb sie keinen Schadensersatz leisten konnten. ${ }^{*}{ }^{8}$ Der verurteilte Herr konnte wählen zwischen Schadensersatz oder Weggabe des Sklaven. Dieses Wahlrecht im Rahmen der Rechtsfolge ähnelt der Haftung des Tiereigentümers bei der actio de pauperie. Die Ursprünge der Noxalhaftung werden allgemeinhin im Racherecht vermutet. Die Auslieferung des Täters sollte dazu dienen, dass das Opfer an ihm Vergeltung üben konnte. ${ }^{*} 39$

\subsection{Rechtssoziologische Sicht}

Stimmen für die Öffnung der Rechtssubjektdefinition finden sich in der Soziologie. Luhmann betrachtet in seiner Systemtheorie soziale Systeme anstelle von menschlichen Individuen, die aus Kommunikationen und deren Zurechnung als Handlungen bestehen. ${ }^{*} 40$ Kommunikation versteht er als Selektionsprozess, bei dem jeweils eine Auswahl hinsichtlich Information, Mitteilung und Verstehen getroffen wird. Erst bei Vorliegen des Verstehens existiere die Kommunikation, die dann zum Bestehen eines Systems führt. Dieses System müsse in der Lage sein, autopoietisch aus Kommunikation weitere Kommunikation zu produzieren. ${ }^{* 11}$ Obwohl Luhmann seine Systemtheorie auf menschliche Individuen in Form sozialer Systeme ausgerichtet hat, lässt sich der Gedanke auf nicht-menschliche Entitäten übertragen. ${ }^{*}{ }^{42}$ Erfüllen autonome Roboter die Anforderung des Kommunikationsbegriffs, so wäre es möglich, sie als soziales System zu verstehen.

Latour hingegen setzt sich direkt mit der Einordnung von nicht-menschlichen Entitäten auseinander und schafft dafür den Begriff des „Aktanten“, um einen Ausweg aus der zwingenden Klassifikation als Subjekt oder Objekt zu schaffen. ${ }^{*} 43$ Durch die Schaffung einer eigenen Kategorie für nicht-menschliche Entitäten soll verhindert werden, dass weder Subjekte verdinglicht noch Objekte vermenschlicht werden. ${ }^{*} 4$

Während Latour den Aktantenbegriff damit auf eine Vielzahl von Objekten ausdehnt, fasst Teubner den Begriff enger und versteht unter Aktanten nur solche nicht-menschliche Entitäten, die in der Lage sind, sich an ihre Umwelt anzupassen. ${ }^{*} 45 \mathrm{Um}$ als (Teil-)Rechtssubjekte anerkannt zu werden, müssten die Aktanten autonom sein. ${ }^{*}{ }^{6}$ Dafür seien drei Voraussetzungen zu erfüllen: Intentionales Handeln, Fähigkeit zur Kommunikation mit der Umwelt und Fähigkeit zur Entscheidung unter Ungewissheit. Da bereits automatisierte Roboter mit der Umwelt kommunizieren sowie zielgerichtet handeln können, seien dies lediglich notwendige, jedoch keine hinreichenden Voraussetzungen. Zur Feststellung der Autonomie sei die Fähigkeit des Roboters zur Entscheidung unter Ungewissheit erforderlich. Diese sei vorhanden, wenn die Entscheidung eines Roboters, der zwei Möglichkeiten zur Auswahl hat, vom Entwickler des Roboters nicht nachvollzogen werden kann und dieser keine Aussagen über den Ausgang einer weiteren Entscheidung in der Zukunft treffen kann. Ist dieses Maß an Autonomie erfüllt, müsse die Gesellschaft dem Roboter eine (Teil-)Rechtsfähigkeit zusprechen, um das damit einhergehende Autonomierisiko zu würdigen. Entwickelt man diesen

37 Rudolf Düll (Fn 29) 1, 5.

38 David Deroussin, Histoire du Droits des Obligations (2. Auflage, Economica 2012), 774.

39 Ibid; Max Kaser (Fn 30) 163-164; Wolfgang Kunkel, Paul Jörs und Leopold Wenger, Römisches Recht: Römisches Privatrecht, Abriß des Römischen Zivilprozeßrechts (3. Auflage, Springer 1978), 269; Milena Polojac (Fn 31) 93; Feliciano Serrao, „La Responsabilità per fatto altrui in diritto romano“ (1963) 66 Bullettino dell'Istituto di Diritto Romano Vittorio Scialoja 19, 29.

40 Dazu Niklas Luhmann, Soziale Systeme - Grundriß einer allgemeinen Theorie (7. Auflage Suhrkamp 1999), 194-240.

41 Niklas Luhmann, Organisation und Entscheidung (3. Auflage VS-Verlag 2011), 388. - DOI: https://doi.org/10.1007/9783-531-93042-8.

42 So auch Gunther Teubner, „Elektronische Agenten und große Menschenaffen: Zur Ausweitung des Akteurstatus in Recht und Politik“ (2006), ZfRSoz, 5, 14f. - DOI: https://doi.org/10.1515/zfrs-2006-0103.

43 Bruno Latour, Das Parlament der Dinge - Für eine politische Ökologie (Suhrkamp 2001), $108 f$.

44 Ibid 114f.

45 Gunther Teubner (Fn 42) 19.

46 Dazu im Folgenden Gunther Teubner, „Digitale Rechtssubjekte? - Zum privatrechtlichen Status autonomer Softwareagenten“ (2018) AcP, 155, 173-177. - DOI: https://doi.org/10.1628/acp-2018-0009. 
Gedanken konsequent weiter, bedeutet dies, dass aus rechtssoziologischer Perspektive die Erhebung des Roboters zu einem Rechtssubjekt durchaus möglich, ja sogar erforderlich ist, wenn ein bestimmtes Maß an Autonomie des Roboters vorliegt.

\subsection{De lege lata}

Im BGB wird systematisch zwischen Rechtssubjekten (natürliche und juristische Personen) und Rechtsobjekten (alle Gegenstände wie Sachen, Tiere und Rechte) unterschieden. Soziologische Ansätze bzgl. der Definition von Subjekten finden im BGB keine Ausprägung. Roboter sind als Sachen de lege lata als Rechtsobjekte einzuordnen. ${ }^{*} 47$ Der moderne Begriff des Rechtssubjekts hat seine Wurzeln vielmehr in den Lehren Kants und Savignys. Nach Kant unterscheidet das Bewusstsein über das „Ich“ sowie die damit einhergehende Würde den Menschen von Sachen und Tieren. ${ }^{*} 8$ Für den Status als Subjekt ist zudem ausschlaggebend, ob die Entität frei hinsichtlich moralischer Gesetze handeln kann. ${ }^{*} 49$ Aus diesen Anforderungen an ein Subjekt ergeben sich hinsichtlich von Robotern grundlegende Fragen: Haben autonome Roboter ein menschliches Bewusstsein? Können autonome Roboter moralisch handeln? ${ }^{*} 50$ Sofern diese Fragen nicht deutlich bejaht werden können, ist aus Kant'scher Perspektive der Subjektstatuts auf Menschen beschränkt und kann nicht Robotern zuteilwerden.

Aus der historischen Perspektive von Savigny zeigt sich, dass die Rechtspersönlichkeit untrennbar mit dem Menschsein verbunden ist, denn Voraussetzung für den Status eines Rechtssubjekts ist der freie Wille. ${ }^{*}{ }^{1}$ Bezogen auf die Gewährung einer Rechtspersönlichkeit für Roboter ergeben sich nach dieser Definition Probleme. Zunächst ist der Roboter nicht menschlich, sondern bleibt ein animiertes, vom Menschen geschaffenes Objekt, egal wie hoch die Ähnlichkeit mit dem Menschen ist. Zudem stellt sich die Frage, ob ein Roboter einen freien Willen haben kann? Dies ist eindeutig nicht der Fall. Ein Roboter existiert nicht eo ipso, sondern wird vom Menschen geschaffen durch Bau und Programmierung. Selbst wenn man dem Roboter das höchstmögliche $\mathrm{Ma} ß$ an Autonomie zugesteht, also seine Entscheidungen nicht vorhersehbar sind, beruht die grundsätzliche Entscheidungsmacht des Roboters auf menschlicher Programmierung, ist also determiniert anstelle von frei. ${ }^{*}{ }^{2}$ Nach Savignys Konzeption erfüllen Roboter trotz ihrer Autonomie nicht die Anforderungen für den Status als Rechtssubjekt.

Schließlich stellt sich die Frage nach den verfassungsrechtlichen Grenzen der Rechtsubjektqualität. Das Grundgesetz ist als objektive Wertentscheidung Maßstab für alle Rechtsgebiete. ${ }^{*} 53$ Es ist durch die über allem stehende Garantie der Menschenwürde in Art. 1 I GG anthropozentrisch ausgerichtet. ${ }^{*} 4$ Daher genießt der Subjektstatus des Menschen besonderen Schutz. ${ }^{*} 5$ Nach einer Ansicht folge daraus die Ausschließlichkeit des menschlichen Subjektstatus. Tiere bspw. könnten hingegen keine Rechte haben. ${ }^{*}{ }^{6} \mathrm{Nach}$ einer anderen Auffassung gewährt Art. 1 GG keinen exklusiven Status des Menschen. ${ }^{*} 57$ Der Schutz der Menschenwürde besagt nur, dass der Mensch nicht zum Objekt gemacht werden darf (sog. Objektformel $\left.{ }^{*} 58\right) .{ }^{*} 59$

\footnotetext{
Steffen Wettig und Eberhard Zehender (Fn 16) 127.

48 Immanuel Kant, Anthropologie in pragmatischer Hinsicht (Dürr 1899), 1.

49 Immanuel Kant, „Einleitung in die Metaphysik der Sitten“ in Königlich Preußische Akademie der Wissenschaften (Hrsg.), Kant's gesammelte Schriften, Band 6 (Georg Reimer 1914), 223.

50 Daran knüpft auch die Frage im Strafrecht nach der Schuldfähigkeit von Robotern an. Siehe dazu ausführlich bei Eric Hilgendorf, „Können Roboter schuldhaft handeln?“ in Susanne Beck (Hrsg.), Jenseits von Mensch und Maschine, (Nomos Verlag 2012), 119-132. - DOI: https://doi.org/10.5771/9783845237527-119; Monika Simmler und Nora Markwalder, „Roboter in der Verantwortung - zur Neuauflage der Debatte um den funktionalen Schuldbegriff“ (2017) ZSTW, 20-47; Gerhard Seher, „Intelligente Agenten als ,Personen“ im Strafrecht?“ in Sabine Gless und Kurt Seelmann (Hrsg.), Intelligente Agenten und das Recht (Nomos Verlag 2016), 45-60. - DOI: https://doi.org/10.5771/9783845280066-45.

51 Friedrich Carl von Savigny, System des heutigen römischen Rechts, Band 2 (1840), 2. - DOI: https://doi.org/10.1515 /9783111443348

52 Siehe zu der Inkompatibilität von freiem Willen und Determinismus auch Peter van Inwagen, The Incompatibility of Free Will and Determinism (1975), Philosophical Studies 27, No 3, 185ff. - DOI: https://doi.org/10.1007/bf01624156.

53 BVerfGE 7, 198; Michael Sachs in Sachs-Kommentar zum GG (8. Auflage, C.H. Beck 2018), Vorb. Abschnitt I, Rn. 32.

54 Christian Hillgruber in Beck'scher Onlinekommentar zum GG (42. Aufl. 2019), Vorb. Art. 1; Matthias Herdegen in Maunz/ Dürig-Kommentar zum GG (90. EL, C.H. Beck 2020), Art. 1 I, Rn. 2.

55 Christian Hillgruber (Fn 54) Art. 1, Rn. 13.

56 Rupert Scholz in Maunz/Dürig-Kommentar zum GG (90. EL, C.H. Beck 2020), Art. 20a, Rn.75.

57 Jens Kersten, „Menschen und Maschinen - Rechtliche Konturen instrumenteller, symbiotischer und autonomer Konstellationen" (2015) JZ 1, 7. - DOI: https://doi.org/10.1628/002268814x14151859100293.

58 st. Rspr: BVerfGE 45, 187 (228); BVerfGE 115, 118 (153); BVerfGE 144, 20 (207).

59 Utz Schliesky, „Digitale Ethik und Recht“ (2019) NJW 3692, 3696.
} 
Mit der Schaffung der juristischen Person wurde von der engen Ausrichtung auf den Menschen abgewichen. Die Abweichung ist nicht so deutlich, wie sie auf den ersten Blick scheint, denn die Stellung der juristischen Person ist eng mit den natürlichen Personen verbunden. Dies zeigt sich einerseits in der Gewährleistung der Bildung von Gesellschaften in Art. 9 I GG, die dazu beitragen soll, dass natürliche Personen ihre Persönlichkeit in organisierten Gruppen entfalten können. ${ }^{* 60}$ Andererseits wird im Rahmen von Art. 19 III GG bei der Anerkennung der Grundrechtsfähigkeit die Lehre vom personalen Substrat herangezogen, bei der die Grundrechtsfähigkeit der juristischen Personen vom BVerfG per Durchgriff auf die dahinterstehenden Menschen rückgekoppelt und den juristischen Personen nicht eo ipso zugesprochen wird. ${ }^{*}{ }^{* 1}$ Allerdings weicht das BVerfG selbst von der Lehre des personalen Substrats ab, da es die Grundrechtsfähigkeit von Stiftungen bejaht, ${ }^{* 62}$ die über kein personales Substrat verfügen, sondern bei denen vielmehr das Stiftungsvermögen im Vordergrund steht. ${ }^{*} 63$

Diese Abweichung von der engen Ausrichtung auf den Menschen lässt das Grundgesetz auch in Bezug auf Tiere zu, indem Art. 20a GG den Tierschutz verfassungsrechtlich gewährleistet und eine ausschließlich anthropozentrische Auslegung des Art. 20a GG nicht zwingend ist. ${ }^{*} 64$ Vielmehr sei der Tierschutz in Art. 20a GG pathozentrisch ausgerichtet. ${ }^{*} 65$ Daraus wird teils abgeleitet, dass es im Rahmen der Verfassung möglich sei, Tieren eine Rechtspersönlichkeit zu verleihen. ${ }^{* 66}$ Bereits vor der Einführung des Art. 20a GG wurde diskutiert, dass Tieren zwar keine Pflichten, jedoch aber Rechte gewährt werden müssten. ${ }^{*} 67$ Alternativ wird Art. 20a GG als bloße objektiv-rechtliche Garantie verstanden, die für Tiere keine eigenen Rechte begründet. ${ }^{* 68}$

Es lässt sich festhalten, dass das Grundgesetz von der absoluten Ausrichtung auf den Menschen Ausnahmen ermöglicht, wenn es soziale, rechtliche oder ökonomische Gründe erfordern. ${ }^{* 69}$ Daher ist auch die Anerkennung von Robotern als Rechtssubjekte im Rahmen des Grundgesetzes trotz seiner anthropozentrischen Ausrichtung möglich und nicht per se verfassungswidrig. ${ }^{*} 70$

\section{Resultat und Ausblick}

Zusammenfassend zeigt dieser Überblick, dass autonome Roboter - basierend auf der Ansicht Savignys de lege lata zu den Rechtsobjekten zählen. Eine Erhebung in den Subjektstatus de lege ferenda wäre nach dem Grundgesetz nach einer Ansicht zulässig und aus rechtssoziologischer Sicht sogar geboten.

Die Frage nach dem rechtlichen Umgang mit autonomen Robotern wird durch den Fortschritt der Technik immer bedeutsamer. Dies hat das Europäische Parlament erkannt und darauf mit seiner Entschließung zur Teilrechtsfähigkeit reagiert. Die Verleihung der Qualität einer E-Person ist de lege ferenda möglich und in Grenzen zulässig. Doch ist die Tragweite der Entscheidung hinsichtlich der Zukunft des unserem Rechtssystems zugrundeliegenden Menschenideals beachtlich. Eine Überhöhung der Sachautonomie stünde in der Traditionslinie der griechischen Antike und dem Glauben an hybride Mischwesen. Davon rückten schon die Römer ab. Die Grenze zwischen Mensch und Maschine sollte weiterhin konstitutiv bleiben.

60 BVerfGE 38, 281 (303); Rupert Scholz in Maunz/Dürig-Kommentar zum GG (92. EL, C.H. Beck 2020), Art. 9, Rn. 11; Wolfram Höfling in Sachs-Kommentar (Fn 53) Art. 9, Rn. 3.

61 St. Rspr: BVerfGE 21, 362 (369f.); BVerfGE 61, 82 (101); BVerfGE 143, 246 (313).

62 BVerfGE 75, 138 (160).

63 Jens Kersten (Fn 57) 7; Birgt Weitemeyer in Münchener Kommentar zum BGB, Band 1 (8. Auflage, C.H. Beck 2018), § 80, Rn. 1.

64 Dietrich Murswiek in Sachs-Kommentar (Fn 53) Art. 20a, Rn. 23.

65 Carolin Raspé, Die tierliche Person (Duncker Humblot 2013), 221. - DOI: https://doi.org/10.3790/978-3-428-53972-7; Johannes Caspar und Martin Geissen, „Das neue Staatsziel ,Tierschutz‘ in Art. GG Artikel 20a GG“ (2002) NVwZ, 913; Dietrich Murswiek (Fn 64) Art. 20a, Rn. 31b; BT-Drs. 14/8860, S. 3.

66 So Jens Kersten (Fn 57) 7. Eine ausführliche Untersuchung zur Frage nach der Subjektstellung für Tiere findet sich bei Saskia Stucki, Grundrechte für Tiere (Nomos Verlag 2016), 173ff. - DOI: https://doi.org/10.5771/9783845271774 (ausgehend vom Schweizer Recht) sowie bei Carolin Raspé (Fn 65) 281ff.

67 Eisenhart v Loeper und Wasmut Reyer, „Das Tier und sein rechtlicher Status: Zur Weiterentwicklung von Transparenz und Konsequenz des Tierschutzrechts“(1984) ZRP 205, 208f.; Heinrich Freiherr von Lersner, „Gibt es Eigenrechte der Natur?“ (1988) NVwZ 988.

68 Rupert Scholz in Maunz/Dürig-Kommentar zum GG (Fn 56) Art. 20a, Rn. 76.

69 Jens Kersten (Fn 57) 7.

70 So auch ibid. 